\title{
EVHA, UN LOGICIEL D'ÉVALUATION DE L'HABITAT DU POISSON SOUS WINDOWS.
}

\author{
V. GINOT* \\ Laboratoire d'Hydroécologie Quantitative, Division Biologie des \\ Ecosystèmes Aquatiques, CEMAGREF, 3 bis quai Chauveau, \\ 69336 Lyon Cedex 09, France.
}

\begin{abstract}
RÉSUMÉ
EVHA est un logiciel destiné à l'évaluation de la capacité physique d'accueil des rivières vis-à-vis de certaines espèces de poissons cibles. Son approche est essentiellement graphique. Après avoir dépouillé les données topographiques et calé le modèle hydraulique, l'utilisateur étudie sa station selon les quatre représentations proposées, courbes fonction du débit, vues en plan, profils en long ou profils en travers. Les variables, Surface Pondérée Utile (SPU), SPU normée à 100 mètres, et valeur d'habitat, mais aussi surfaces mouillées, hauteurs d'eau, vitesses et nombres de Froude, volumes, peuvent être calculés soit en restant au niveau du point d'observation, soit en moyennant les valeurs par transect, par faciès, ou pour l'ensemble de la station. Les données générées peuvent être visualisées sous forme textuelle et exportées au format des principaux tableurs pour un traitement plus complet. Développé sous Windows, EVHA gère le «copier-coller», la sauvegarde des graphes au format vectoriel, l'impression et la gestion de la couleur et du noir et blanc. Sa bibliothèque de courbes de préférences peut s'enrichir des courbes produites par l'utilisateur.
\end{abstract}

\section{EVHA, A WINDOWS SOFTWARE FOR FISH HABITAT ASSESSMENT IN STREAMS.}

\section{ABSTRACT}

EVHA is devoted to the simulation of fish habitat in streams. It essentially uses a graphical approach. EVHA includes modules for the validation of topographic data and for the calibration of the hydraulic model. After dealing with these, the user considers the habitat or physical variables according to the four representations proposed : discharge curves, maps, cross sections, and longitudinal profiles. The text of the calculated data can be read on separate Windows, and both graphs and data can be saved in Windows environment, through output files compatible with standard software or through the clipboard. Three habitat variables, the Weighted Usable Area (WUA), the $100 \mathrm{~m}$ normalized WUA, and the habitat value, but also wetted surfaces, depth, velocity and Froude number can be evaluated for each measurement point or averaged to the scale of the cross section, of the morphological unit, or of the whole site. User's preference curves can be employed beside EVHA's ones.

\section{INTRODUCTION}

Lors des réflexions engagées autour de la loi de 1984 relative à la pêche en eau douce, la Direction de l'Eau du Ministère de l'Environnement et la Direction Etudes et Recherche d'EDF ont demandé au CEMAGREF de faire l'inventaire des méthodes susceptibles de répondre quantitativement à la question des débits réservés. Parmi ces

\footnotetext{
*Adresse actuelle : Institut de Limnologie de Thonon-les-Bains, INRA, BP 511, 74203 Thonon Cedex, France.
} 
méthodes, le CEMAGREF a retenu et testé sur 12 rivières de référence françaises I'IFIM ou Instream Flow Incremental Methodology développée par I'U.S. Fish \& Wildlife Service de Fort Collins au début des années 1980 (STALNAKER, 1979 ; BOVEE, 1982), et connue en France comme la méthode des microhabitats (SOUCHON et al., 1989). Mais la mise en application effective dans notre pays de cette méthode, déjà très utilisée outre-Atlantique (REISER et al., 1989), devait encore passer par le développement d'un support informatique. C'est, nous l'espérons, chose faite avec EVHA.

Nous ne donnons ici que les principes de la méthode. Pour plus de détails, notamment sur ses limites d'application, le lecteur pourra se référer aux trois articles cités ci-dessus, ou aux guides méthodologique et informatique accompagnant le logiciel.

\section{PRINCIPE DES MODÉLISATIONS BIOLOGIQUE ET HYDRAULIQUE}

Le modèle biologique employé par EVHA est donc dérivé de celui de I'US Fish \& Wildlife Service. L'aire d'étude est partitionnée en petites surfaces homogènes, appelées cellules, pour lesquelles les trois variables physiques d'habitat, hauteur d'eau, vitesse et substrat, sont observées ou calculées par le modèle hydraulique. Une cellule est délimitée par deux points d'observation successifs sur un transect et leurs projections sur les limites de représentativité amont et aval du transect. Des "courbes de préférences" sont définies pour chaque espèce de poisson subdivisée en stades de développement. Elles donnent la note d'affinité, entre 0 et 1 , du stade considéré vis-à-vis des trois variables définissant l'habitat. Chaque cellule est pondérée par le produit de ces trois notes d'affinité, et est ensuite agglomérée à ses voisines par transect, par faciès, ou pour l'ensemble de la station pour donner la Surface Pondérée Utile (SPU) du secteur vis-à-vis du stade de l'espèce considérée. Répété pour chaque débit, on obtient une courbe qui permet de se faire une idée de l'impact d'une modification du débit sur la capacité physique d'accueil de la station étudiée vis-à-vis de l'espèce considérée. La SPU est souvent normalisée à 100 mètres de linéaire de cours d'eau $(\mathrm{SPU} / 100 \mathrm{~m})$ pour favoriser les comparaisons entre rivières. On peut également s'affranchir des surfaces, et calculer la valeur d'habitat qui est le rapport de la SPU à la surface mouillée. A l'échelle de la cellule, la valeur d'habitat est le produit des trois notes d'affinité.

Le modèle hydraulique est de type fluvial : la ligne d'eau est calculée en remontant de section hydraulique en section hydraulique depuis le bas de la station. Dans les rares cas où une zone de la station se trouverait en régime torrentiel, EVHA calcule la ligne d'eau dite critique. EVHA utilise la formule de Limérinos, validée par HEY (1982), pour le calcul de perte de charge linéaire. Comparativement à celle plus classique de Manning, cette formulation tient compte de la résistance frontale à l'écoulement des éléments les plus grossiers, ce qui permet de garder un coefficient de frottement constant, même à bas débit, sur des rivières où certains éléments du substrat peuvent atteindre la hauteur d'eau. La ligne d'eau est calculée en interpolant systématiquement 10 sections hydrauliques temporaires entre deux sections réelles. II a été écrit à partir des programmes Talweg et Fluvia de BAUME et POIRSON (1984).

Les vitesses, hauteurs d'eau et substrats nécessaires aux calculs d'habitat sont relevés le long de sections perpendiculaires à la rivière appelées transects. Une procédure particulière permet de calculer des vitesses pour chacun de ces points d'observation, le modèle hydraulique, monodimensionnel, ne donnant que la vitesse moyenne sur le transect.

\section{TRAVAILLER SOUS EVHA}

Le travail sous EVHA se fait en deux phases : la construction du projet et l'analyse des résultats.

La construction du projet commence par le dépouillement topographique des données. Le contrôle en est essentiellement graphique : vue en plan, profils en travers et profil en long s'associent aux avertissements et commentaires du programme de dépouillement (figure 1). L'utilisateur doit ensuite caler le modèle hydraulique en se basant sur les observations de terrain. Pour cette étape délicate, EVHA propose une procédure de calage automatique et restitue les résultats avec une série de diagnostics sauvegardés sous forme de fichier texte. 


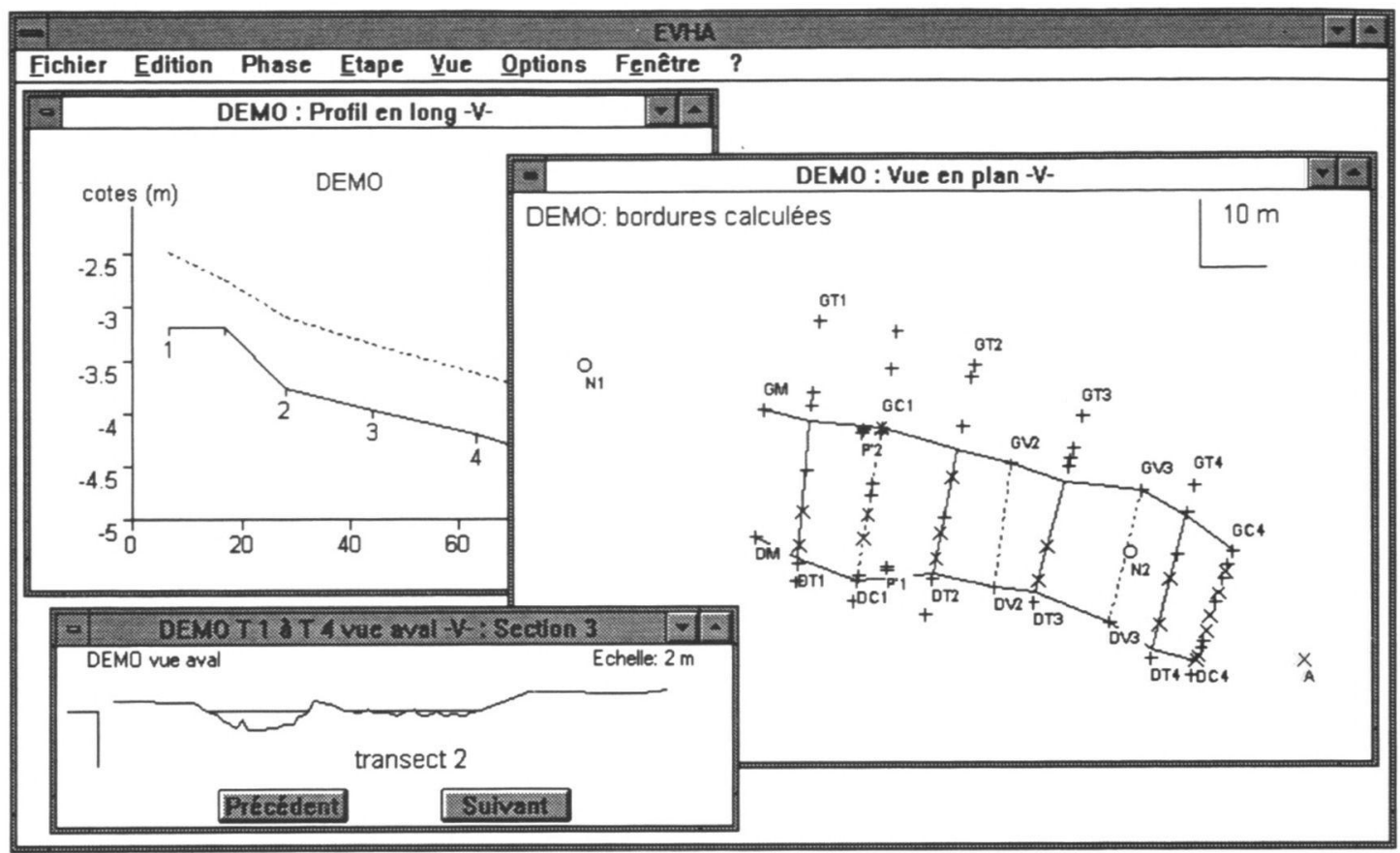

Figure 1 : La construction du projet comporte trois étapes : l'initialisation, le dépouillement topographique des données et le calage hydraulique du modèle. Le contrôle du déroulement de ces opérations se fait par la visualisation de la station dans les trois dimensions de l'espace. L'affichage des codes des points cartographiés facilite la recherche des erreurs.

Figure 1 : The first step when working with EVHA consists in the validation of the topographic data and the calibration of the hydraulic model. This process is controlled by three-dimensional graphical display of the station. Plotting the identification code of the topographic points is of great help for error location.

L'analyse des résultats n'est par contre pas guidée par EVHA, bien qu'il soit toujours proposé des options par défaut. Elle est essentiellement graphique. L'utilisateur dispose de quatre types de représentation, les courbes fonction du débit, les profils en long, les profils en travers, et les vues en plan. Le principe est que, pour chaque graphe, il soit possible d'afficher les données générées. Tout graphe et tout fichier de données associé peut ensuite être sauvegardé, au format vectoriel Windows Metafile pour les graphes et au format ASCII des principaux tableurs pour les données, ou exporté vers un autre logiciel via le presse papier. L'utilisateur peut également utiliser ses propres courbes de préférence si elles sont validées pour le type de cours d'eau qu'il étudie.

Un document de travail important est, bien sûr, la courbe de capacité d'accueil en fonction du débit (figure 2). L'utilisateur sélectionne sa variable d'habitat, puis un ou plusieurs stade(s) de poisson(s). S'il n'a sélectionné qu'un stade d'un seul poisson, il peut y ajouter les trois composantes de la variable d'habitat, c'est-à-dire la valeur obtenue si la hauteur, la vitesse, ou le substrat agissaient seuls. Cela permet de comprendre comment évoluent les composantes de la capacité d'accueil en fonction du débit. L'utilisateur choisit aussi la gamme et le nombre de débits à figurer, et l'échelle de restitution des résultats : transect par transect, pour l'ensemble de la station, ou par faciès définis comme des suites contigües de transects. 


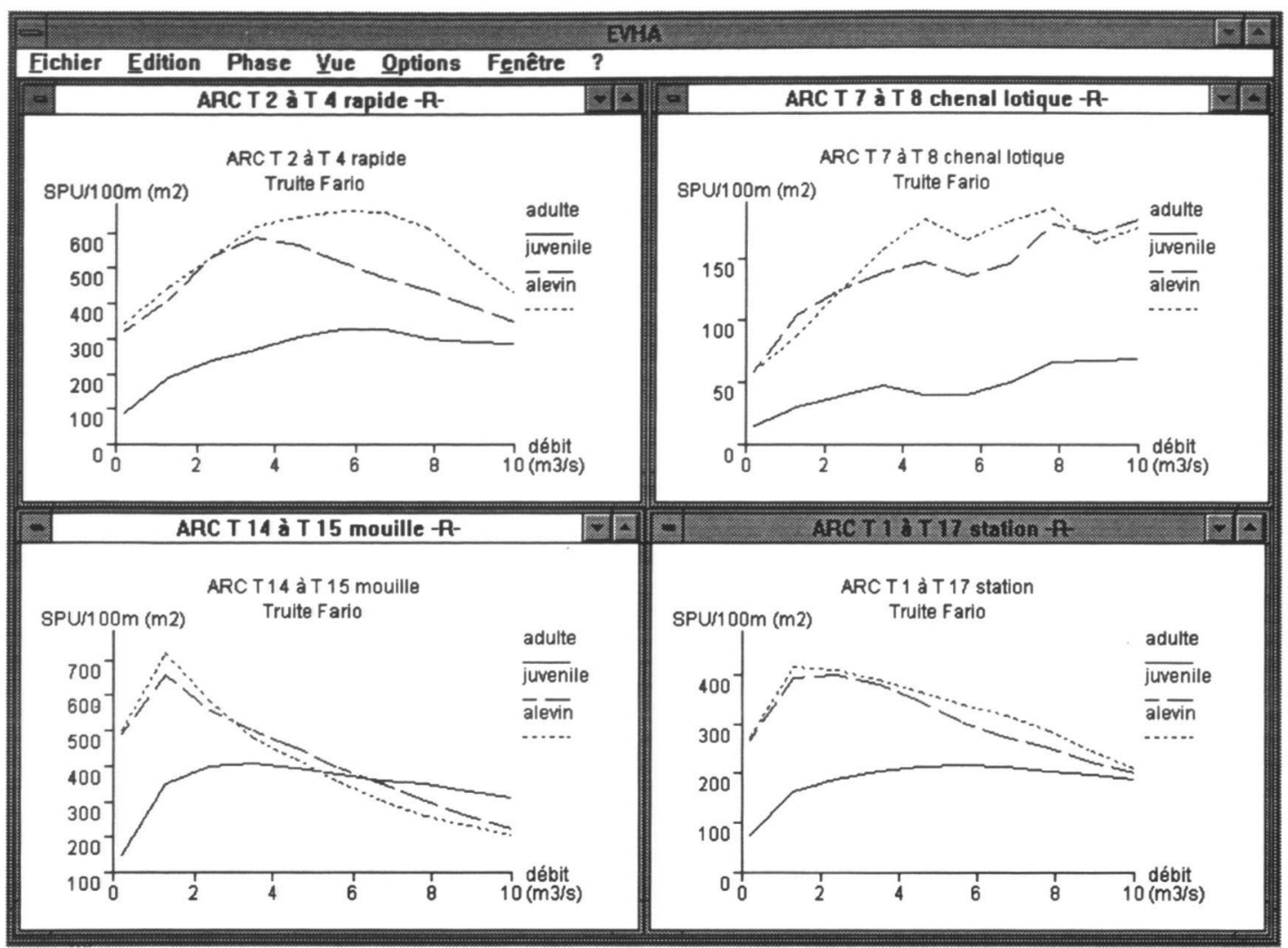

Figure 2 : Courbes d'habitat en fonction du débit. Sur cet exemple comportant 17 transects, l'utilisateur a défini 3 faciès à comparer à la moyenne de la station, sélectionné 10 débits dans une gamme de 0,2 à $10 \mathrm{~m}^{3} / \mathrm{s}$, et choisi 3 stades de l'espèce truite. Les faciès ont souvent des comportements très différents en fonction du débit.

Figure 2 : Habitat versus discharge curves. For this site described with 17 cross sections, the user has defined 3 morphological units for comparison with the mean of whole area. He has selected 10 discharge values ranging from 0,2 to $10 \mathrm{~m}^{3} / \mathrm{s}$, and chosen to consider 3 trout stages. On such curves, morphological units often show quite different patterns according to the discharge.

Les trois autres types de représentation permettent d'affiner le résultat et de générer des données qui pourront servir à des calculs complémentaires (figure 3 ) :

- Les profils en travers, sur lesquels la seule variable d'habitat visualisable est la valeur d'habitat puisqu'il n'y a pas ici de notion de surface. S'y ajoutent les lignes d'eau, la vitesse et le nombre de Froude. Les données sont ici relatives aux points d'observation, contrairement aux vues en plan où les données sont relatives aux cellules.

- Les profils en long permettent de visualiser les lignes d'eau, les trois variables d'habitat (SPU, SPU/100 m et valeur d'habitat), ainsi que la surface mouillée (normée ou non à 100 mètres), la vitesse et le nombre de Froude, les largeurs et sections mouillées, les volumes. Cinq courbes sont superposables sur un même graphe, correspondant à cinq débits différents.

- Les vues en plan permettent de visualiser la valeur d'habitat (ainsi que ses trois composantes), la vitesse, la hauteur d'eau ou le nombre de Froude. Les données sont relatives aux cellules et le fichier correspondant donne également la valeur des hauteurs, vitesses, substrats et surfaces en plus de la variable demandée. 


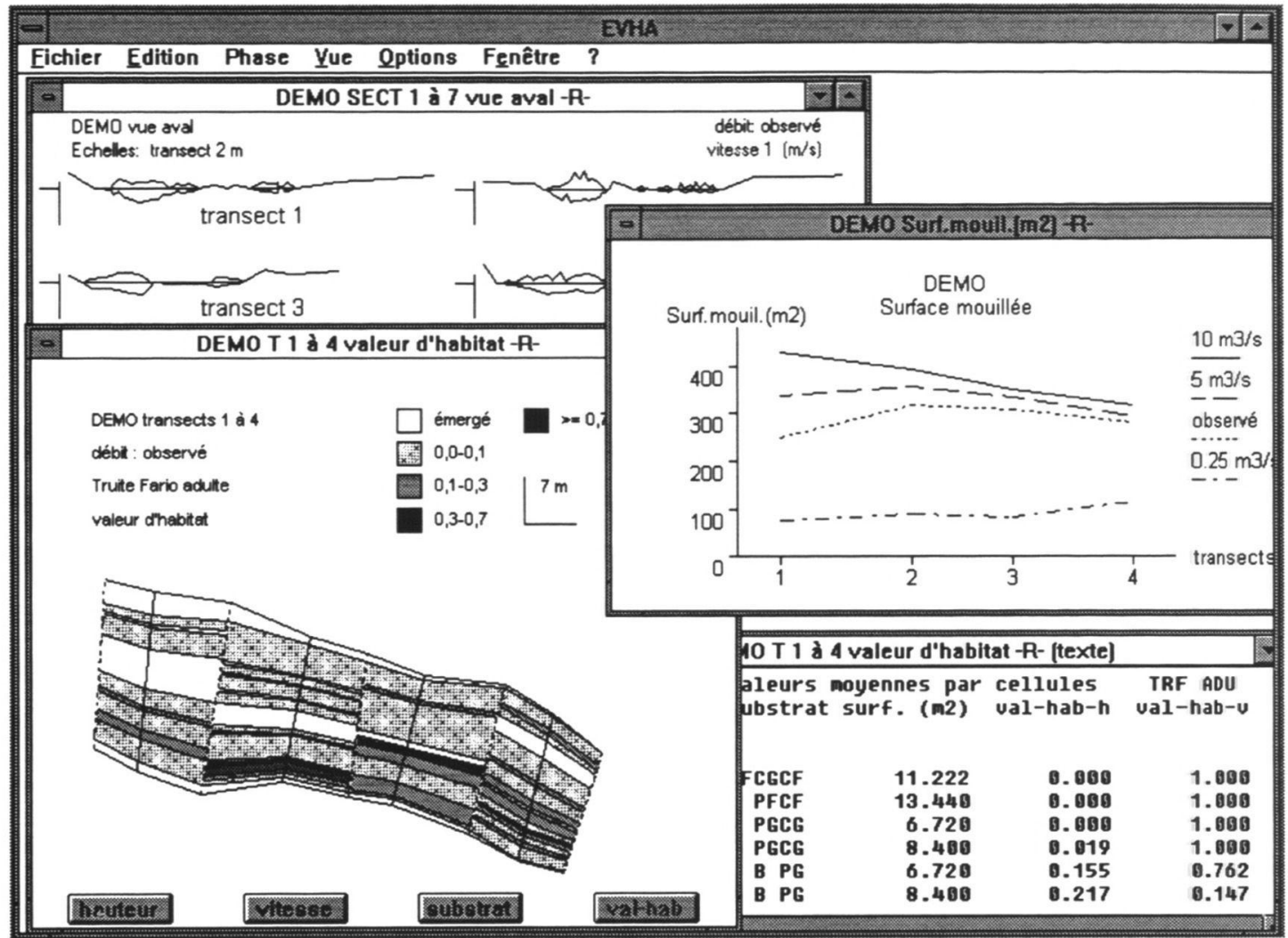

Figure 3 : Trois types de représentations physiques viennent en complément des courbes fonction du débit. L'utilisateur a choisi ici de figurer les vitesses au débit observé sur les profils en travers (option tous les profils dans la même fenêtre), les surfaces mouillées pour quatre débits sur le profil en long, et la valeur d'habitat en vue en plan. On notera pour cette dernière les trois boutons permettant d'accéder aux trois composantes de la variable d'habitat. A chaque graphe est associé un fichier de données que l'on peut visualiser, sauvegarder, ou exporter (ici les valeurs relatives à la vue en plan).

Figure 3 : Three kinds of representation help the user to understand habitat versus discharge curves. Here, the user has chosen to draw cross-sections with the velocities observed, Froude numbers for three discharges along the longitudinal profile, and a map of the habitat value for adult trout. In the latter graph, three push-buttons allow to switch between the three constitutive elements of habitat value. An ASCII data file is associated with each graph. It can be observed, saved, and exported.

\section{CONFIGURATION INFORMATIQUE}

EVHA se contente de toute configuration supportant Windows 3.1 ou Windows 95 , mais il est conseillé de disposer d'un 486 DX ou d'un Puntium doté de 8 à 16 Mo de mémoire vive et d'un écran couleur 17 pouces pour tirer pleinement partie de ses possibilités graphiques.

EVHA peut supporter jusqu'à 50 sections hydrauliques (soit 24 transects hydrobiologiques), chaque section pouvant comporter 100 points de mesures. 10 positions peuvent être utilisées pour la lunette de visée et le nombre maximum de points topographiés est de 500 . 


\section{CONCLUSION}

L'avenir nous dira si EVHA est le logiciel que tout le monde attendait pour appliquer sereinement la méthode des microhabitats sur petits et moyens cours d'eau. Nous pensons cependant qu'il permet d'obtenir rapidement et presque ludiquement les résultats essentiels, tout en apportant les informations nécessaires à la bonne compréhension de ces derniers.

L'utilisateur ayant accès à toutes les données générées via les différentes représentations graphiques, EVHA ne se limite pas à la méthode des microhabitats stricto sensu : tout travail ayant besoin d'une information hydraulique fine sur les hauteurs, vitesses et substrat, mais aussi sur les variables qui en dérivent, ou sur les surfaces et les volumes occupés par la rivière, peut utiliser les résultats d'EVHA. Dans cet ordre d'idées, la prochaine version pourrait inclure une boîte à outils permettant à l'utilisateur, après avoir effectué ses propres calculs sur les données générées par EVHA, de visualiser ses résultats selon les quatre types de représentation proposés.

Pour terminer, nous ne saurions trop recommander à l'utilisateur de bien étudier le guide méthodologique associé et de respecter les protocoles de prise de mesures et les conseils pour le rendu des résultats : ils sont là pour aider le futur lecteur à comprendre la genèse des résultats et à se convaincre de leur pertinence.

\section{REMERCIEMENTS}

Ce logiciel est le résultat d'un programme de recherche de l'équipe d'Hydroécologie Quantitative du CEMAGREF de Lyon : Yves SOUCHON, Hervé CAPRA, Marc POUILLY et Sylvie VALENTIN développent les aspects biologiques, efficacement complétés sur le terrain par Pascal ROGER, Michel PHILIPPE et bien des collègues. Vincent GINOT et Christian BOUDEAU ont réalisé le portage sous Windows. Merci également à ceux qui ont maintenant quitté l'équipe, Francis TROCHERIE co-initiateur du projet, Elisabeth FRAGNOUD et Jean-René MALAVOI. Le développement informatique d'EVHA fait l'objet d'un financement du Ministère de l'Environnement, Direction de l'Eau.

\section{BIBLIOGRAPHIE}

BAUME J.P., POIRSON M., 1984. Modélisation numérique d'un écoulement permanent dans un réseau hydraulique maillé à surface libre, en régime fluvial. La Houille Blanche, 1/2, 95-100.

BOVEE K.D., 1982. A guide to stream habitat analysis using the Instream Flow Incremental Methodology. Instream Flow Information Paper $n^{\circ} 12$, FWS/OBS 82/26, Western Energy and Land Use Team, U.S. Fish and Wildlife Service, Fort Collins, Colorado, $248 \mathrm{p}$.

HEY H.D., 1982. Design equations for mobile gravel-bed rivers. In : HEY R.D., BATHURST J.C., THORNE C.R. (eds.), Gravel-Bed Rivers, John Wiley and Sons Ltd, 553-580.

REISER D.W., WESCHE T.A., ESTES C., 1989. Status of instream flow legislation and practices in North America. Fisheries, 14 (2), 22-29.

SOUCHON Y., TROCHERIE F., FRAGNOUD E., LACOMBE C., 1989. Les modèles numériques des microhabitats des poissons : application et nouveaux développements. Revue des sciences de l'eau, 2, 807-830.

STALNAKER C.B., 1979. The use of habitat structure preferenda for establishing flow regimes necessary for maintenance of fish habitat. In WARD and STANFORD (eds.), The Ecology of Regulated Streams, 326-337, Plenum Press, New-York. 